\title{
GENE ASSORTMENT AND PREFERENTIAL MATING IN THE BREEDING OF GERMAN FANCY CATS
}

\author{
ROY ROBINSON \\ St Stephen's Road Nursery, Ealing, London, W.13
}

Received 19.v.69

\section{INTRODUCTION}

ONE of the problems confronting practical genetics of the domestic cat is that of obtaining adequate data to demonstrate the expected ratios of assorting mutant genes. It is not unknown for published papers to contain numerical data which would almost certainly be held to be unsatisfactory had the animal been one of the common laboratory species. Essentially, the problem is two-fold. The domestic cat is a relatively expensive animal to breed and rear under laboratory conditions. It is also relatively slowbreeding, so that to obtain large numbers, the economic aspects often become disproportionately onerous. Little can be done to offset this unless the genetic information can be derived as an adjunction to some other function of cat breeding.

\section{The material}

In several countries of the world, the breeding of pedigree cats is exceedingly well organised, comprising societies to nurture specific breeds and the provision of official standards of perfection. Great store is made of pedigree breeding and the matings of individual animals are carefully regulated. Now, breeds of cats contain almost all of the known colour mutants, and, collectively, the breeding of pedigree cats constitutes a considerable genepool. It may be wondered if this potential source of breeding data could perhaps be drawn upon.

Unfortunately, certain difficulties have to be overcome. Although details of pedigree cats are registered with central bodies, the registration is usually for individual cats and, generally, it is impossible to obtain information on complete litters. However, there is an exception in the case of one country. The controlling body in the German fancy cat (Der I. Deutschen Edelkatzenzuchter-Verbandes $e$. V.) requires that entire litters be registered. This information is periodically published in their Fachzeitschrift, "Die Edelkatze", together with details of parentage. This policy commenced in the autumn of 1963. In this study, records from vol. 13, no. 4 (1963), to vol. 18, no. 4 (1968) have been consulted.

Over the years, a considerable quantity of data has accumulated and prima facie would seem open to analysis. A certain amount of caution is necessary, however, in the too ready acceptance of the results at their face value. For instance, there could be wastage in the form of kittens which died before registration. A check on the mean litter size for the various breeds indicated that this may occur but, if so, it is on a small scale. The distribution of litter sizes, as published, compare favourably with those obtained from other sources. The main reason for this is undoubtedly the individual care and attention which are lavished on the cats. Also, if there 
is much non-registration, with one exception to be discussed later, it is non-selective in that the majority of gene ratios, which emerged under analysis, are not distorted from expectation.

Pedigree cat breeding as a whole is not a homogeneous entity. Barriers exist between certain breeds, in as much as these are never or rarely crossed. On the other hand, within certain breeds or groups of breeds, there is extensive crossing of colour types. Gene assortment can be detected and these have in fact yielded useful confirmatory data on known and conjectured modes of inheritance. Furthermore, it may be wondered just how far the concept of quasi-random mating may hold. By this term, it is meant that, although cat breeders confine their animals and plan their pairings, there is no co-ordination between breeders, and their actions are independent. It has been possible to examine this question and to show that a degree of preferential mating is apparently exercised by breeders for an important sector of the fancy.

\section{Mutant genes investigated}

One of the primary purposes of study is to glean data on the assortment of certain coat colour genes to augment the meagre amount of data currently available in the literature. The following mutant genes and phenotypes will feature in the analysis:

\begin{tabular}{cl} 
Gene symbol & \multicolumn{1}{c}{ Phenotype and remarks } \\
$a$ & Non-agouti or self-black \\
$b$ & Brown or chocolate \\
$c^{\text {ch }}$ & Silver or smoke \\
$c^{\prime}$ & Siamese \\
$d$ & Blue dilution \\
$W$ & Dominant white; either yellow, blue or odd-eyed \\
$O$ & Orange; sex-linked.
\end{tabular}

A detailed account of the above mutants, as well as a general discussion of cat genetics, may be found in Robinson (1959). The sex-linked gene $O$ was formerly known as yellow $(y)$ but has been changed for reasons of consistent nomenclature (Committee on Standardized Genetic Nomenclature for cats, 1968).

\section{(a) New data}

\section{The analysis}

The main data are arranged in tables 1 and 2. The expectations in the right-hand half of table 1 represent a preliminary approximation to the observed litters and take cognisance of the fact that, with this kind of data, those litters which should have produced phenotypically different young but failed to do so by chance, will not be included. This aspect can be allowed for by the use of the formula $2 \cdot-1 /\left(2^{\prime}-1\right)$ and $4^{\prime-1} /\left(4^{*}-3^{\prime}\right)$ for the backcross and $\mathrm{F}_{2}$ generations, respectively; $s$ being the number of kittens per sibship. The $\chi^{2}$ values in the last column of the table do not reflect this comparison but are based on the procedure described by Bailey (1961; section 14.31) for analysing assortment of genes in this sort of situation. These $\chi^{2}$ have one degree of freedom. 
a. The origin of non-agouti is lost in antiquity; the black cat is probably one of the oldest mutants known in domestic animals. Despite this, the amount of reliable data on the heredity of $a$ is scanty. Whiting (1918) records a mating of agouti $\times$ agouti which gave five tabby and one black.

TABLE 1

Assortment data for mutant coat colour genes of the domestic cat

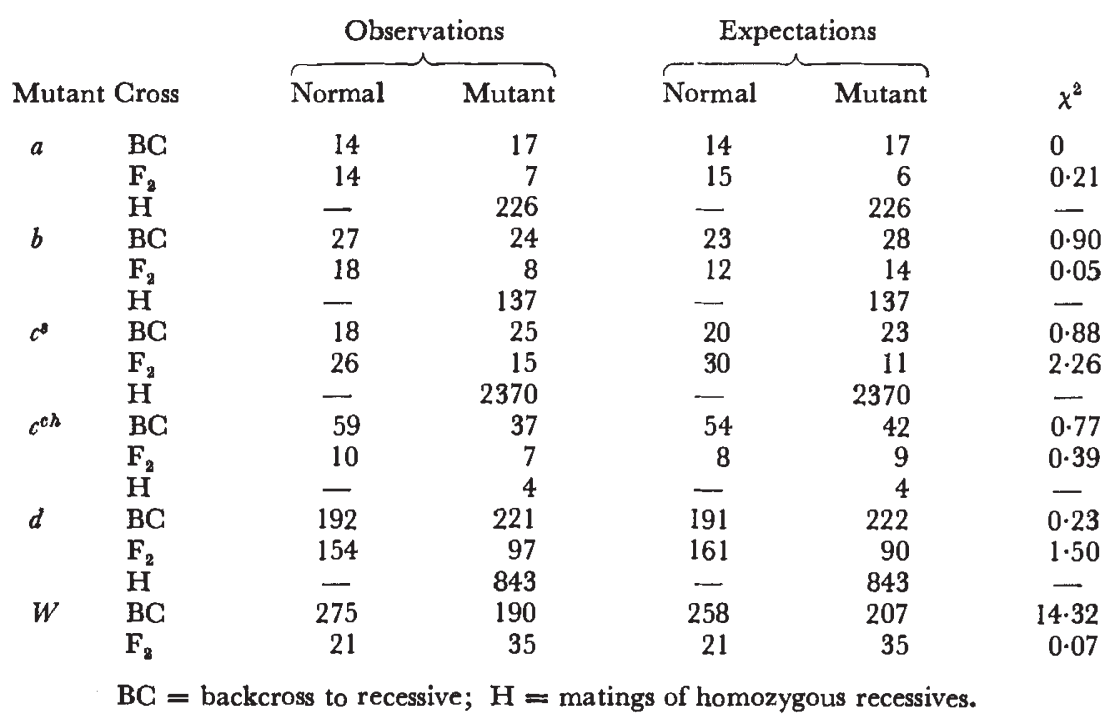

Todd (1963) records the segregation of agouti versus black in a backcross ( 3 out of 8 offspring). The data of table 1 for $a$ adds considerably to these results and confirms that the gene is inherited as a recessive. Being recessive, matings of non-agouti inter se produce only non-agouti and the figure of 226

TABle 2

Assortment of the sex-linked $\mathrm{O}$ gene in the domestic cat

\begin{tabular}{|c|c|c|c|c|c|c|c|c|}
\hline \multicolumn{2}{|c|}{ Cross } & \multirow{2}{*}{$\begin{array}{l}\text { No. of } \\
\text { pairings }\end{array}$} & \multicolumn{2}{|c|}{ Males } & \multicolumn{3}{|c|}{ Females } & \multirow[b]{2}{*}{$\chi^{2}$} \\
\hline Female & Male & & or & $+r$ & $O O$ & $O+$ & ++ & \\
\hline$O O$ & or & 37 & $47(53)$ & - & $56(53)$ & - & $\ldots$ & 0.47 \\
\hline$O+$ & or & 93 & $75(75)$ & $66(75)$ & $76(75)$ & $81(75)$ & - & 1.57 \\
\hline$O+$ & $+r$ & 101 & $72(85)$ & $106(85)$ & - & $76(85)$ & $86(85)$ & $8 \cdot 13$ \\
\hline$O O$ & $+r$ & 26 & $27(29)$ & - & - & $30(29)$ & - & 0.03 \\
\hline++ & or & 28 & - & $34(34)$ & - & $33(34)$ & $\ldots$ & 0.02 \\
\hline
\end{tabular}

non-agouti is evidence of this. Moreover, these represent self-black and self-blue cats about which there cannot be ambiguity. In addition, the majority of Siamese cats are non-agouti $\left(a a c^{8} c^{8}\right)$; breeding true to type and available as supplementary evidence is necessary.

$b$. The existence of a brown-coloured cat was described as long ago as 1931 by Bamber and Herdman. Unfortunately, no experimental work could be performed with the animal. A chocolate-point Siamese has been bred by cat fanciers for many years and Ullman and Hargreaves (1958) state that a self-brown cat could be obtained by crossing with an ordinary 
non-agouti and inbreeding the $\mathrm{F}_{1}$. However, still no data are forthcoming; until Todd (1963) contributed a small amount which are incidental to other observations. These consist of the assortment of 4 brown out of 8 young for a backcross of black heterozygotes $F_{1}$ to brown.

During the whole of this time, $b$ has been assumed to be inherited as a recessive to normal, largely by analogy with the proven recessive heredity of comparable brown genes in other mammalian species. There has been no reason to doubt this assumption and confirmation is now provided by the data relating to the $b$ gene in table 1 . The assortment ratios are in agreement with the inheritance of a recessive trait.

Those matings of black with brown which gave only black progeny will not yield useful information except where the number is large enough to suggest that the black parent is homozygous. However, the totality of such matings must be considered if the results are to be correctly assessed. The total number of segregating and non-segregating sibships is 30; of which 19 were non-segregating. Out of these, one had a total progeny of 14 black young, one had 9 , one had 7 , while one had 6 young. The sibship of 14 young is probably by itself reasonably conclusive that black is dominant to brown while the other families provide complementary evidence.

$d$. Doncaster (1904) came to the conclusion that blue dilution was inherited as a recessive to normal pigmentation. This seems to be the case, although Doncaster's results are inclined to be vague. Whiting (1918) published ample data to show that dilution is inherited as a monogenic character, but these could not discriminate between dominant or recessive heredity. Fortunately, blue cats are popular and are freely interbred with black in the long-haired (Perser) breeds and in the Siamese. Consequently, considerable data could be found, as shown by the table. The breeding of blue animals from black parents is decisive in establishing recessive inheritance.

$c^{c h}$. The chinchilla-like mutant in the cat is known as silver and information on the mutant is given by Keeler and Cobb (1933) and Thompson et al. (1943). All of the data is consistent with the hypothesis that normal, silver and Siamese constitute an allelic series. Again, the crucial data on the mode of inheritance of $c^{c h}$ relative to normal is small, comprising five normal and one silver from normal parents. In the fancy cat, silver may be found in three breeds: smoke $a a c^{c h} c^{c h}$, silver $c^{c h} c^{o h}$, and chinchilla $c^{o h} c^{c h}$.

The data on $c^{c h}$ in table 1 are based on the smoke (mainly) and silver phenotypes as regards assortment, and these indicate that smoke and silver are inherited as recessive to normal. Additionally, three matings of chinchilla $\times$ chinchilla gave 15 chinchilla offspring. Unfortunately, fancy records can give no information on the allelism of $c^{c h}$ and $c^{s}$ because breeds carrying the two genes differ rather sharply and would never be interbred in the normal course of pedigree breeding.

$c^{8}$. In contradiction to other mutants, there is a fair amount of data on the heredity of $c^{8}$ (Tjebbes, 1924; Keeler and Cobb, 1933; 1936; Thompson et al., 1943; Moutschen, 1950; Little, 1957; Todd, 1963). In spite of this, data on the critical breeding of Siamese offspring from type parents are provided only by Tjebbes (an $\mathrm{F}_{2}$ of 7 type and 2 Siamese). There is no real doubt, however, that $c^{8}$ does not behave as a recessive to normal, if only because similar mutants in other species are inherited as recessive. The results given by table 1 fully confirm the recessive mode of inheritance. 
$W$. Two forms of dominant white exist in the cat. A piebald form (symbolised as $S$ ) and an all-white form (except for ocassional spots of colour on the head), usually with yellow eyes but also with blue eyes or even odd-eyed (symbolised as $W$ ). Both forms are inherited as dominant to type and it has been proposed that the two genes are alleles. However, this proposal has yet to be established and it is more convenient to treat $S$ and $W$ as representing distinct loci. Although not large, the amount of data on $W$ is sufficient to demonstrate that the gene is dominant to type (Whiting, 1918; Tjebbes, 1924; Robinson, 1959; Todd, 1966a).

The present data confirm the dominance of $W$ but also reveal a marked excess of type segregants. The extent of the excess is obscured by the crude presentation of the expectation shown by the table. The proportion of type segregants is estimated to be $57 \pm 2 \cdot 6$ for the backcross and $26 \pm 7 \cdot 5$ for the $\mathrm{F}_{2}$. The departure from the espected 25 per cent. for the $\mathrm{F}_{2}$ is negligible. However, the departure from the expected 50 per cent. for the backcross is highly significant. Accepting the reported data at their face value, this can only mean that the $W+$ cat is sub-viable compared with type. The $W+$ animal has a greater propensity to die either ante-natally or post-natally during the period between birth and registration. A tentative estimate of the viability of $W+$ is 75 per cent. (or a 25 per cent. chance of death for any cause). This seems to be rather a high mortality, yet it would require a sample of about 100 to detect it. This is too large a number for the subviability to be detected in ordinary cat breeding unless one was particularly on the look-out.

It may be wondered if an examination of mean litter sizes could throw light on the deficiency of $W$ animals. The mean litter size is $3.17 \pm 0.11$ kittens for the backcross ( 162 litters) and $2 \cdot 88 \pm 0.25$ for the $F_{2}$ (27 litters). These means may be compared with a mean of $3.05 \pm 0.06$ based upon 534 litters of long-haired non-white breeds. The long-haired breeds are compared because $W$ occurs in the fancy combined with the long-haired character. The above figures give no support to the idea that the mean litter size of white-producing litters is below average. Only the $F_{2}$ has a low value and this is not significant. All of these means appear unusually low, however, and this may be a confusing factor. In the same German fancy data, for example, the mean for the Siamese breed is $3 \cdot 81 \pm 0.07$. All of the above means are significantly below this mean and also of a "theoretical mean" of 4 kittens which is the generally accepted average litter for the cat. It seems probable that some general influence is reducing the litter size in the long-haired breeds.

Jones (1922) has investigated the extent of foetal degeneration in the cat and has made a comparison of the relative amount of degeneration in normal coloured and in white cats (the latter defined as an individual with less than 10 per cent. of coloured fur). He could not establish a significance in mean litter sizes for the two groups ( 4.48 versus 4.08 , respectively) but he could do so for the proportion of foetal degeneration (3.84 versus 10.8 per cent. respectively). It is concluded that white cats have a higher rate of foetal loss. These observations are performed on ordinary domestic cats for New York and it is known that white cats are rare in this population (Todd, 1966b). It follows, therefore, that the unknown sires are probably coloured and the matings are effectively backcrosses. Jones suggests that the white gene may have "a lethal action of some sort" even when 
heterozygous. Jones' observations would support the idea that $W+$ cat is subviable in comparison with normal.

An alternative explanation could be, of course, that not all of the white cats are being registered by German breeders. Could there be a special reason for this? A possibility exists in the deafness of some blue-eyed white cats. If these are detected, it may be that these animals are quietly discarded. It is impossible to ascertain if this could be happening or, if so, whether to such an extent to produce a significant deficiency of $W$ animals. In Germany, there is no bar to the registration of deaf white cats, hence, if non-registration is a cause, it is due to the ethical principles of breeders.

\section{(b) Sex-linkage and non-random mating}

The orange gene manifests $X$ borne sex-linkage and relatively extensive data has been published (Doncaster, 1913; Bamber and Herdman, 1927, 1932). Even so, the data arranged in table 2 represent an appreciable addition. The assortment analysis can be applied directly to the data since all five genotypes are distinguishable. Phenotypically, the genotypes $O X$ and $O O$ are orange, $O+$ tortoiseshell (normal and orange patch-work) while $+X$ and ++ are normal. The main body of the table gives the distribution of offspring for the pairings shown in the left-hand column while the figures in brackets represent the expected frequencies rounded off to the nearest whole number. The $\chi^{2}$ values on the right-hand column have appropriately one or three degrees of freedom. All are non-significant except for the value of $8 \cdot 13$ (d.f. $=3$ ) for the pairing $O+\times+r$. The high value of $\chi^{2}$ is due to an excess of normal coloured males. The reason for this is obscure. Misclassification could scarcely be an important factor. The value of 8.13 is only just beyond the 5 per cent. level of significance, however, so the excess is probably due to chance.

The segregation of $O$ could yield information on the extent that independent action of individual breeders approximate to random mating. Two approaches are possible. One is by consideration of the relative frequencies of the six phenotypes among the kitten population and the other is consideration of the frequencies of the six different parental matings. The basic assumption is that the observed frequencies will agree with the expected frequencies as derived from the observed proportion of $O$ genes. Now, these approaches cannot be applied mechanically. The fancy cat population consists of a number of distinct breeds, some of which are interbred and some of which are not. For instance, the long-haired breeds would not be crossed with the Siamese or similar breeds. The reason for this is that the body conformation differs sharply, and is inherited polygenically, so that first-cross offspring would be neither of one breed nor the other.

The $O$ gene does not feature prominently in breeds other than the longhaired, so attention will be focussed on these. Preliminary examination of the kitten population and pairing frequencies indicate that within the longhaired breeds, part of the population is not involved in crosses with $O$ carrying animals. Consequently, it was decided to reject the whole of the type $\times$ type pairings and to concentrate on those pairings in which at least one parent carried an $O$ gene. In fact, these are the 285 pairings of table 2 . The rejection of the $++x++$ pairings meant that the usual equilibrium proportions for a sex-linked gene in a panmictic population has to be 
modified. The five phenotypes and their expectations are now (writing $D=$ $\left.6(1-q)+2 q^{2}\right):$

$\begin{array}{lccccc}\text { Phenotypes } & \text { Or } & +r & \text { OO } & O+ & ++ \\ \text { Expectation } & \frac{1}{D} & \frac{(2-q)(1-q)}{D} & \frac{q}{D} & \frac{2(1-q)}{D} & \frac{(1-q)^{2}}{D} \\ q=0.479 & 242 & 192 & 116 & 252 & 66 \\ \text { Observation } & 223 & 206 & 132 & 230 & 76\end{array}$

The proportion $(q)$ of the $O$ gene was found by maximum likelihood iteration and is $0.479 \pm 0.02$. Fitting this value into the expectations and multiplying by 867 gives a succession of expected frequencies which may be compared with those observed. The $\chi^{2}$ test for agreement with expectation is 8.16 ( 3 degrees of freedom) a value which just exceeds the 5 per cent. level of significance.

Consideration of pairing frequencies reveals that only a portion of the $++x++$ matings are part of the six possible pairings involving the $O$ gene; hence it was deemed advisable to reject the whole of these from the analysis. The expectations for the five remaining pairings are $\left(D=3-3 q+q^{2}\right)$ :

$\begin{array}{lccccc}\text { Pairing } & O O \times O Y & O+\times O Y & O+\times+r & O O \times+r & ++\times O Y \\ \text { Expectation } & \frac{q^{2}}{D} & \frac{2 q(1-q)}{D} & \frac{2(1-q)^{2}}{D} & \frac{q(1-q)}{D} & \frac{(1-q)^{2}}{D} \\ q=0.481 & 36 & 79 & 86 & 40 & 43 \\ \text { Observation } & 37 & 93 & 101 & 26 & 28\end{array}$

The proportion of the $O$ gene was found as before and is $0.481 \pm 0.021$. Fitting this value into the expectations gives a series of expected frequencies which can be compared with those observed. The $\chi^{2}$ test for agreement between the two sets of figures is 15.23 (3 degrees of freedom), a highly significant value. Thus, there is poor agreement. Apparently, pairing between the various parental phenotypes is not entirely at random although there is seemingly a large random component, since the two sets of figures show a similar pattern. However, there appears to be a preference in the part of breeders to keep tortoiseshell females as against $O O$ or ++ females. A preference which seems to be in the region of 18 per cent. These $O+$ females can be mated to either $O Y$ or $+Y$ males but there seems to be no bias for choice of male.

A feature of the two approaches is that both produce a similar value for the proportion of $O$ genes. The closer approach of the phenotypes in the kitten population to random mating may be spurious and is occasioned by the fact that the mean litter size for the different pairings are unequal. The mean litter sizes for the five pairings, reading from top to bottom (see table 2), are $2 \cdot 8,3 \cdot 2,3 \cdot 4,2 \cdot 2$ and $2 \cdot 4$, respectively. Equalising the mean litter size for each pairing results in a greater departure from random mating (and possibly in a change in the value of $q$ but this was not checked). The two last pairings seem to have smaller litters than the others and for no obvious reason. Breeders have no cause to omit certain details of their litters for these particular pairings. Whatever the cause, it has not seriously distorted the expected assortment ratios for the $O$ gene (table 2). 


\section{5. $X X X$ AND $X O$ fEMALES?}

There is evidence, in the form of unexpected results, either for missclassification of certain phenotypes involving gene $O$ or for irregularities of $X$ chromosome transmission. It is probable that all of the aberrant results can be interpreted as errors of missclassification (unintentional or otherwise) but the possibility of $X$ chromosome irregularities cannot be lightly discounted. The misclassification is revealed by the occurrence of phenotypes among the offspring which are incompatible with expectation for the stated parentage.

Three representative examples are as follows: a pairing of two longhaired tabby cats produced progeny of $6 O r, 5+r, 4 O+$ and $10++$. Now, a long-haired tabby tortoiseshell with a minimum of orange areas could easily be mistaken for an ordinary tabby. Therefore, it may be argued that the mother has been incorrectly classified but is breeding as a tortoiseshell. The second example is that of a long-haired blue female mated to an orange, producing $1 O Y, 2+r, 1 O O$ and $2 O+$. These progeny would be explicable if the blue mother is a blue-cream (dilute tortoiseshell), with the minimum of cream areas, plus the added confusion of the long hair. The third example concerns the young from a mating of a long-haired cream with a cream male, producing $5 O Y, 1+Y, 1 O O$ and $1 \mathrm{O}+$. These are the results to be expected if the cream female was genetically a blue-cream, with the minimum of blue areas which are obscured by the long coat. This is the reverse situation to those of the previous two examples. Seven other similar cases are observed but these produced so few young that detailed consideration is not worth while.

It is possible, however, that the above females may owe either their exceptional phenotype (or breeding behaviour) to an odd karyotype. They could, for instance, be one of a variety of complex karyotypic mosaics or chimeras such as briefly reviewed by Malouf, Benirschke and Hoefnagel (1967). Specifically, $X X Y / X Y$ tortoiseshell males have been detected karyologically. The patch-work pattern of the tortoiseshell does vary within quite wide limits but not normally to complete type or complete orange. However, there is one situation which could possibly bring this about. Suppose the soma of an individual was predominantly $X X X$ and the either complete or partial inactivation of two of the $X$ chromosomes is at random. The two possible genotypes to produce a tortoiseshell will be either $\mathrm{OO}+$ or $O++$ (since one $O$ and one + must be present). Now, with random inactivation of individual chromosomes, the $\mathrm{OO}+$ animal could be almost completely orange while the $O++$ could be almost completely type, because of the two-thirds " built-in " bias towards orange or type as the case may be. In combination with the usual variation exhibited by tortoiseshells, this could be enough to induce a phenotype of 100 per cent. of one colour. There is, of course, no reason to think that the mosaicism need be confined to the soma although it may well be.

Not all cases of misclassification need occur for adult cats whose breeding record belies their phenotypes. Kittens may be incorrectly classified and, in some instances, it may be difficult to decide where the error resides. Eleven cases of misclassification for matings of both $O O x+Y$ and $++\times O Y$ are noted in which exceptional normal or orange young, respectively, are recorded. All eleven are females and it may be held that these represent cases of misclassification of tortoiseshell. At least, this explanation would 
not be easy to disprove. However, another intriguing possibility deserves to be borne in mind. As Searle (1968) points out, the production of these exceptional offspring could be indicative that the mother is $X O$; an anomalous sex chromosome constitution known to be viable in some mammalian species (e.g. house mouse) but not, as yet, for the cat.

For the cat female to be a suspected $X O$, orange females $\times$ normal males should give normal daughters and normal females $\times$ orange males should give orange daughters. The exceptional data for the first mating consists of four normal daughters while that for the second mating consists of one normal and six orange daughters. It should be noted that the exceptional orange young only occur for the second mating which is in full accord with the $X \mathrm{O}$ possibility. Furthermore, four of the orange young were born to one mother and two to another. This could be interpreted either as further support for the $X O$ karyotype or to a propensity for certain parents to produce orange daughters with minimal type areas. The same two parents also produced seven sons of expected phenotypes but no tortoiseshell daughters. This latter aspect could indicate that the exceptional young are misclassified tortoiseshells since tortoiseshell daughters should occur even if the mother is $X O$. The significance of these observations is that exceptional inheritance of the $O$ gene could reveal abnormal $X$ chromosome transmission or karyotype constitutions which would otherwise not be noticed. A karyological examination would then be desirable.

\section{Summary}

1. Breeding results reported by German pedigree cat breeders are analysed for assortment of mutant genes and for departure from quasirandom mating.

2. Data are presented for the first time to show that the $b$ gene is inherited as an autosomal recessive.

3. Additional data are obtained to confirm that autosomal recessive inheritance of $a, c^{c h}, c^{s}$ and $d$, and the autosomal dominance of $W$.

4. Extensive data are obtained on the sex-linked inheritance of $O$. The heredity of $O$ for the long-haired breeds indicate that mating is not entirely at random as far as the orange character is concerned. German breeders show a partiality to retain tortoiseshell females $(O+)$ in preference to orange $(O O)$ or type $(++)$.

5. Aberrant breeding results for the transmission of the $O$ gene infer the possibility of rare $X X X$ and $X O$ chromosome females.

Acknowledgments.-I thank Dr Rosemarie Wolff for directing my attention to the breeding results published in Die Edelkatze and to Dr J.S. Gale for advice on the statistical analysis.

\section{REFERENCES}

BAILEY, N. J. J. 1961. Introduction to the Mathematical Theory of Linkage. London: Oxford University Press.

BAMBER, R. C., AND HERDMAN, R. C. 1927. The inheritance of black, yellow and tortoiseshell coat colour in cats. F. Genet., 18, 87-89.

BAMBer, R. C., AND herdman, E. C. 1931. Two new colour-types in cats. Nature, 127, 558.

BAMBER, R. C., AND HERDMAN, E. C. 1932. A report on the progeny of a tortoiseshell male cat, together with a discussion of his genetic constitution. F. Genet., 26, 115-128. COMMTTEE ON STANDARDISED NOMENCLATURE FOR CATS. 1968. $\mathcal{F}$. Hered., 59, 39-40. 
DONCASTER, L. 1904. On the inheritance of tortoiseshell and related colours in cats. Proc. Camb. Phil. Soc., 13, 35-38.

DONCASTER, L. 1913. On sex-limited inheritance in cats and its bearing on the sex-limited transmission of certain human abnormalities. 7. Genet., 3, 11-23.

JONES, E. E. 1922. The genetic significance of intra-uterine sex ratios and degenerating foetuses in the cat. F. Hered., 13, 237-239.

LITTLE, c. c. 1957. The yellow Siamese cat. J. Hered., 48, 57-58.

KEELER, G. E., AND COBB, v. 1933. Allelomorphism of silver and Siamese coat variations in the domestic cat. F. Hered., 24, 181-184.

KeEler, C. E., AND COBB, v. 1936. Siamese-Persian cats. 7. Hered., 27, 339-340.

MALOUF, N., BENIRSCHKE, K., AND HOEFNAGEL, D. 1967. XX/XY chimerism in a tricolour male cat. Cytogenetics, 6, 228-241.

Moutschen, J. 1950. Quelques particularités hereditaires du chat siamois. Nat. Belges, 31, 200-203.

robinson, R. 1959. Genetics of the domestic cat. Bibliogr. Genet., 19, 273-362.

SEARLE, A. G. 1968. Comparative Genetics of Coat Colour in Mammals. London: Logos Press. THOMPSON, J. C., COBB, V. C., KEELER, C. E., AND DMYTRYK, M. 1943. Genetics of the Burmese cat. F. Hered., 34, 119-123.

TJEBbes, K. 1924. Crosses with Siamese cats. 7. Genet., 14, 355-366.

TODD, N. B. 1963. Independent assortment of Manx and three coat colour mutants in the domestic cat. 7. Hered., 54, 266.

TODD, N. B. 1966a. The independent assortment of dominant white and polydactyly in the cat. 7. Hered., 57, 17-18.

TODD, N. B. $1966 b$. Gene frequencies in the cat population of New York City. 7. Hered., 57, 185-187.

ulliman, E. Von, AND hargreaves, A. 1958. New coat colours in domestic cats. Proc. Zool. Soc. Lond., 130, 606-609.

whiting, P. w. 1918. Inheritance of coat colour in cats. 7. Exp. Zool., 25, 539-570. 